\title{
Multiple Choices of Reactor Core Nuclear Design for ACP100's Application in Different Scenarios
}

\author{
Wang Liangzi, Ju Haitao, Li Qing, Qin Dong, Wang Lianjie, Yu Yingrui, Ning Zhonghao, \\ Wang Chenlin, Guo Rui, Wang Shuai, Zhang Bin, Xiang Hongzhi, Lou Lei and Sun Wei
}

Science and Technology on Reactor System Design Technology Laboratory, Nuclear Power Institute of China. NO. 328 Section 1 Changshun Ave, Shuangliu District, Chengdu, Sichuan

lwon-1z@163.com,jht0@163.com,819712236@qq.com, mcd2264@126.com, yuyr78@163.com,958968105@qq.com,461473283@qq.com, guorui7814@sina.com, wangshu ainpic@163.com, jsszwtzb@163.com, xhz807460944@163.com, 371682011@qq.com, sunwei2005011750@126.com

\begin{abstract}
ACP100 NPP designed by CNNC (China National Nuclear Corporation) is a $125 \mathrm{MWe}$, multi-purpose small modular reactor based on pressurized water reactor technology; it adopts the integrated reactor technology. Different application scenarios bring up different design requirements: some require high compactness, but others care more about a longer cycle length, and some may require a fully mature and conservative design; thus, multiple design choices need to be proposed. Also, the same and most important thing cared by all users is that, the design needs to be validated to satisfy the current nuclear safety standards, and lower cost would be always preferred. Core nuclear design is a key part of the whole NPP design. Basically, nuclear design target of ACP100 is to achieve a reasonable good balance during longer cycle length, larger discharge exposure for fuel assemblies, and maximally using the mature technologies, and of course, with sufficient reactivity control ability for safety assurance. Aiming at satisfying all these different needs maximally, a strategy of supplying multiple nuclear design choices is proposed for ACP100: choice 1. Boron-free plan, this is a compact design with no need for chemistry and volume system, no need for da ily boron adjustment and relative waste storage; choice 2 . Boron and rod co-controlled plan, this is similar with large commercial PWRs, with a lower power peak factor and suitable for broad location sites. Both choices load 57 units of the same type fuel assemblies CF3S (with height reduced from CF3 fuel assemblies) per cycle, and both adopt partial reload and shuffle fuel management strategy to achieve larger discharge exposure. Gd is loaded in the fuel rods in both choices to help control reactivity. Choice 1 loads much more control rod clusters than choice 2, and of course, reactivity adjustment and compensation during operation is totally different between them. Using suitable and reliable software to simulate $t$ he core, through large amount of optimization, both choices achieve a 24-month fuel cycle length; the average discharge exposure of fuel assemblies reach about $40000 \mathrm{MWd} / \mathrm{tU}$, which is competitive among SMRs, especially for boron-free ones; and sufficient reactivity control ability and safety margin is validated to fully meet the reactor safety requirements.
\end{abstract}

KEYWORDS: CNNC, ACP100, SMR, Core Nuclear Design, Multiple Choices 


\section{INTRODUCTION}

ACP100 meets CNNC's (China National Nuclear Corporation) technical development route for small mo dular reactors for multiple utilities. The overall technical orientation of ACP100 is content to the specifica tions and requirements of the 3rd generation nuclear energy system. ${ }^{[1]}$

Nuclear Steam Supply System (NSSS) of ACP100 is designed by Nuclear Power Institute of China (NPI C), it applies integrated system; the reactor power is designed to be $385 \mathrm{MWt}$, contains $57 \mathrm{CF} 3 \mathrm{~S}$ (designed by NPIC, which belongs to CNNC) square fuel assemblies (FAs), core equivalent diameter is $183.19 \mathrm{~cm}$, height of the active core is $215 \mathrm{~cm}$. Major technical parameters of ACP100 are listed in Table I.

Main requirements for nuclear design of the core include: a) enough safety margin is strictly required, dedicated by reasonable low power peak factors and enough shutdown margin; b) discharge exposure of the FAs should be limited under a validated line; c) fuel cycle length should satisfy the overall aim, 24 months, and bestly achieve a good balance with the averaged discharge exposure.

Then, the core design mainly contains three parts: fuel and core loading, reactivity control, fuel cycle design. To meet the multiple application target of ACP100, multiple core design strategy is proposed, both of them satisfy the main requirements mentioned in the last paragraph. The main difference is the reactivity control strategy: one depends on control rods (CRs) only, the other one depends on CRs and soluble boron. Then, the fuel loading, power flattening strategy and even fuel cycle strategy are all different.

Table I.: Major Technical Parameters of ACP100

\begin{tabular}{|l|l|}
\hline Parameters & Value \\
\hline Reactor type & Integral PWR \\
\hline Coolant/moderator & Light water/light water \\
\hline $\begin{array}{l}\text { Thermal/electrical capacity, } \\
\text { MW(t)/MW(e) }\end{array}$ & $385 / 125$ \\
\hline Primary circulation & Forced circulation \\
\hline System pressure, MPa & 15 \\
\hline Core inlet/exit temperatures, ${ }^{\circ} \mathrm{C}$ & $286.5 / 319.5$ \\
\hline Linear power density, W/cm & 116 \\
\hline $\begin{array}{l}\text { Optimal estimation flow rate, } \\
\mathrm{m}^{3} / \mathrm{h}\end{array}$ & 10000 \\
\hline $\mathrm{F}_{\mathrm{Q}}$ design limit & 2.6 \\
\hline $\mathrm{F}_{\triangle \mathrm{H}}$ design limit & 1.6 \\
\hline Fuel enrichment, $\% \mathrm{wt}$ & $\leqslant 4.95$ \\
\hline
\end{tabular}

\section{CORE NUCLEAR DESIGN STRATEGIES AND MAIN RESULTS}

Maximally using mature technology is an important consideration through ACP100's whole design, the core physics design would not be exceptional. $17 \times 17$ height-reduced square fuel assembly $\mathrm{CF} 3 \mathrm{~S}$ (designed and fabricated by CNNC) is chosen for ACP100, because the full-height CF3 has been already applied in the large commercial NPP's design, and many necessary experiments have been finished for CF3 assemblies. In CF3S, $\mathrm{UO}_{2}$ is loaded in cylindrical fuel pellets; the enrichment is limited to be lower than 5\%wt. CRs are composed of mature material Ag-In-Cd; burnable poison is $\mathrm{Gd}_{2} \mathrm{O}_{3}$ that is mixed into the fuel pellet, the content of $\mathrm{Gd}_{2} \mathrm{O}_{3}$ is limited to $10 \% \mathrm{wt}$. 


\subsection{Core Loading}

For both control choices, 57 assemblies are loaded in the core as shown in Figure 1. For the first cycle, lower enrichment fuel is chosen. Compared with large PWRs, the reactor is much smaller, makes radial power flatten more difficult, especially for the rod controlled core. Therefore, for the first cycle, highleakage loading pattern is chosen, and for the following cycles until equivalence, partial low-leak loading pattern strategy is used.

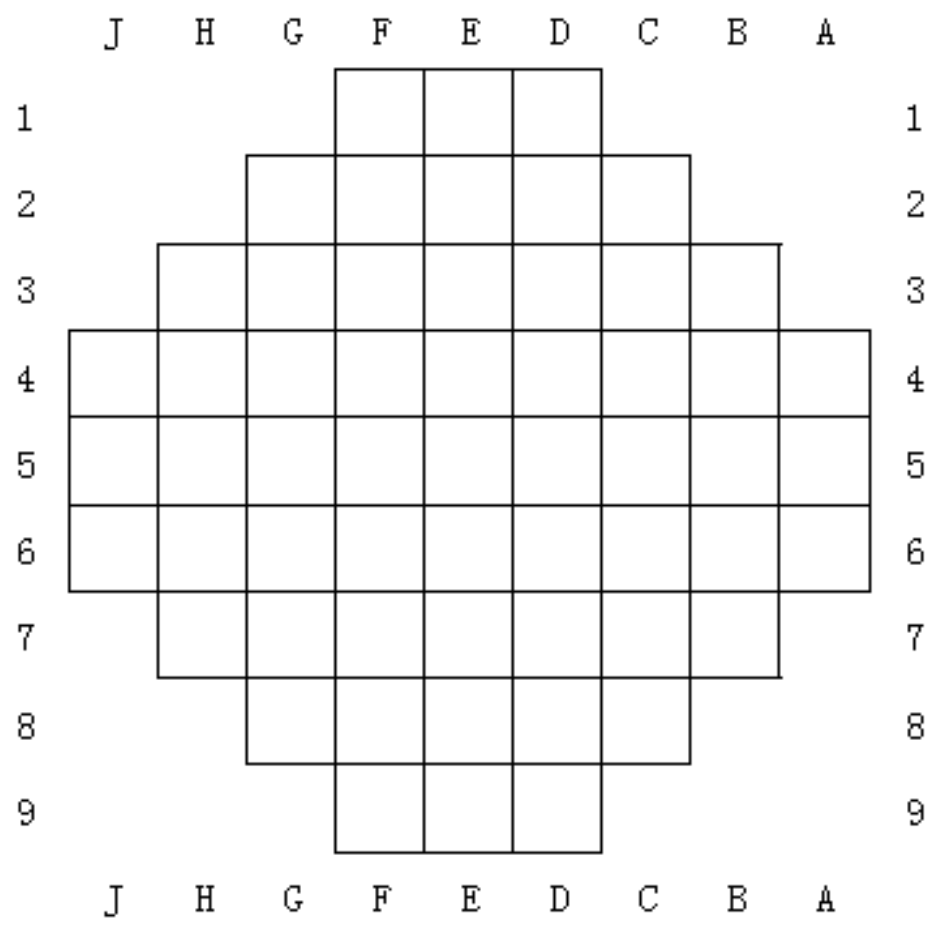

Figure 1. Core Loading Pattern of ACP100.

\subsubsection{Boron-Free Core}

Because there is no soluble boron in the moderator, CRs and burnable poison contributes total reactivity adjustment and compensation during burnup process. Because of the strong neutron absorption of CRs, power distribution heterogeneity is big, and it varies closely with the movement of CRs. To maintain sufficient safety margin, power distribution peak factor is strictly limited based on safety analysis. Complicated loading strategy is necessary to satisfy the limits.

$\mathrm{Gd}_{2} \mathrm{O}_{3}$ mixed in fuel pellets, act as neutron absorber and also used to flatten power distribution. Through differential loading of ${ }^{235} \mathrm{U}$ by enrichment radially in different assemblies, axially in different heights; and differential loading of $\mathrm{Gd}_{2} \mathrm{O}_{3}$ by content in different assemblies and different heights; the power distribution is effectively controlled. There are totally two types of $\mathrm{UO}_{2}-\mathrm{Gd}_{2} \mathrm{O}_{3}$ fuel rods, enrichment of ${ }^{235} \mathrm{U}$ are all $2.5 \% \mathrm{wt}$, with content of $\mathrm{Gd}_{2} \mathrm{O}_{3} 7 \%$ or $10 \%$. There are 5 types of axially differential loading rods: $1.9 \% \mathrm{wt}$ and $3.1 \% \mathrm{wt}$ enrichment fuel is loaded in the first cycle, $3.1 \% \mathrm{wt}, 3.9 \% \mathrm{wt}$ and $4.95 \% \mathrm{wt}$ enrichment fuel is loaded in following cycles. 


\subsubsection{Boron and CR Co-Controlled Core}

For boron and CR co-controlled design, most CRs are always withdrawn from the core during usual operation, only few CRs would be inserted into the core with limited fraction. Boron in the moderator plays key role to compensate the reactivity change. Taking advantage of the homogeneous distribution of boron in the moderator, it's easy for this design to achieve good power flatten feature.

Therefore, it is very different from the boron-free core, this core only loads one type of $\mathrm{Gd}_{2} \mathrm{O}_{3}$ content, $8 \%$; and no axial division needed to be used. The power distribution is achieved by differential reactivity loading in different assemblies.

\subsection{Reactivity Control}

\subsubsection{Boron-Free Core}

As is shown in Fig.2, 'CR' means a cluster of control rods is loaded in the relative assembly. 49 CRs are loaded among totally 57 assemblies. AIC is the neutron absorber in CRs, which is a mature and cheap material. When the reactor operates at nominal power, parts of the CRs are inserted and the other ones are withdrawn. Other than CRs, some $\mathrm{UO}_{2}-\mathrm{Gd}_{2} \mathrm{O}_{3}$ fuel rods are loaded in the core to help reactivity control.

As the burnup of fuel and burnable poison, CRs position varies to compensate the reactivity variation of the core. Because CRs distributes heterogeneously in the core, the power distribution is very sensitive to CRs radial and axial positions. Therefore, this design leads to a higher power peak factor, and makes a compression to the safety margin. This may bring up additional safety system request. At the same time, doing efforts to flatten power distribution in the core is necessary.

As is mentioned in 2.1.1, complicate LP (loading pattern) of fuel and poison is used to achieve this. On the other hand, CRP (Control Rods Procedure) decides the movement sequences of CRs, and which part of the fuel is nearby of strong neutron absorbers is then decided. So, CRP affects the power distribution strongly. As LP and CRP are deeply interconnected, this design takes large amount of time and computer source to do optimization. Finally, with the variation of loading from first to equivalence cycle, the CRP design varies too.

\subsubsection{Boron and CR Co-Controlled Core}

For this design, reactivity control is much easier than the former one. Soluble boron acid in the moderator, control rods and $\mathrm{Gd}$ poison contribute together. $224 \mathrm{UO}_{2}-\mathrm{Gd}_{2} \mathrm{O}_{3}$ rods are loaded in the first cycle, 160 $\mathrm{UO}_{2}-\mathrm{Gd}_{2} \mathrm{O}_{3}$ rods are loaded in the equilibrium cycle. About $1700 \mathrm{ppm}$ soluble boron in moderator is needed in the beginning of cycle to assist reactivity control, as the fuel burning up, the boron concentration decreases as well. Fig. 3 shows CRs loading in the core. Only few CRs are inserted slightly for reactivity adjustment, most CRs are withdrawn during operation, the key function of which is to bring negative reactivity quickly under the shutdown request.

As a neutron absorber, soluble boron distributes in the core almost homogeneously, thus, it is easy to hold good power distribution. Large safety margin is then created, so it's possible to increase the power density or to reduce the requirements for safety systems. 
However, since the boron concentration is manually adjusted; reactor chemistry and volume system is necessary, requires a larger space compared with the rod controlled one; and large amount of waste water would come out, this is a big burden during the reactor operation.

\begin{tabular}{|c|c|c|c|c|}
\hline $\mathrm{CR}$ & $\mathrm{CR}$ & $\mathrm{CR}$ & $\mathrm{CR}$ & $\mathrm{CR}$ \\
\hline $\mathrm{CR}$ & $\mathrm{CR}$ & $\mathrm{CR}$ & $\mathrm{CR}$ & \\
\hline $\mathrm{CR}$ & $\mathrm{CR}$ & $\mathrm{CR}$ & $\mathrm{CR}$ & \\
\hline $\mathrm{CR}$ & $\mathrm{CR}$ & $\mathrm{CR}$ & & \\
\hline $\mathrm{CR}$ & & & & \\
\hline $\mathrm{E}$ & $\mathrm{D}$ & C & B & A \\
\hline
\end{tabular}

Figure 2. CR Loading Pattern of the Boron-Free Core

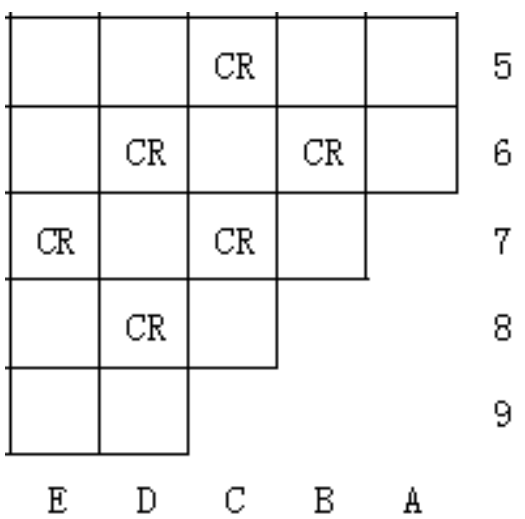

Figure 3. CR Loading Pattern of the Boron and CR Co-Controlled Core

\subsection{Fuel Management Design}

The key target of this part is to achieve 24 months cycle length, and simultaneously, keep the maximum discharge burnup under $52000 \mathrm{MWd} / \mathrm{tU}$. Usage of mature fuel and burnable poison is preferred, so fuel enrichment should be lower than $5 \%$ wt.

To achieve a maximum utilization of fuel, multi-batch loading strategy is applied in both design choices; fuel management analysis has been completed mainly about batch numbers, assembly number per batch, enrichment and the loading pattern. For the Co-controlled core, the analysis method is similar to big commercial PWRs. For the boron-free one, since LP and CRP are deeply interconnected, fuel management affects LP, and so need to be analyzed with CRP; this increases the analysis input dimension and leads to tremendous amount of design analysis. 
Small reactor has a larger radial flux gradient, which makes the power distribution more difficult to be optimized; absolute low leakage, high leakage and partial low leakage loading pattern are all analyzed and the conclusion is that, partial low leakage loading pattern would provide a good balance between power distribution and fuel utilization.

The analyzing results show that ${ }^{[2]}$, matching the cycle length and the discharge limit, a 3-batch strategy is preferred. To achieve the 24 months target, the number of every new FAs batch should not lower than 20 , and the average enrichment of the core needs to be high enough. Associated other considerations like keeping enough power flatten and shutdown margin, the fuel management strategy is finally optimized to:

1) 3-batch reloading, with 20-24 FAs per batch;

2) Mixed enrichment fresh FAs are loaded;

3) Partial low leakage loading pattern.

Figure 4 shows the loading pattern of the equilibrium cycle for the co-controlled core, Table II and Table III show more detailed usage of FAs loading for every cycle. Figure 5 shows the pattern for the boronfree core, Table IV and Table V show more detailed information of this core.

Even the detailed design is different for two cores, with the same FA type, discharge limits, and same cycle length, the strategy both comply with the three features mentioned above.

\begin{tabular}{|c|c|c|c|c|}
\hline 3 & 2 & 1 & 3 & 1 \\
\hline 2 & 3 & 2 & 1 & 2 \\
\hline 1 & 2 & 2 & 1 & \\
\hline 3 & 1 & 1 & & \\
\hline 1 & 2 & & & \\
\hline $\mathrm{E}$ & D & C & B & A \\
\hline
\end{tabular}

1: Fresh assembly; 2: Reloaded assembly from N-1 cycle; 3: Reloaded assembly from N-2 cycle.

Figure 4. Loading Pattern of ACP100 (1/4 core)

\begin{tabular}{|c|c|c|c|c|}
\hline 2 & 2 & 2 & 1 & 1 \\
\hline 2 & 3 & 2 & 1 & 3 \\
\hline 2 & 2 & 2 & 1 & \\
\hline 1 & 1 & 1 & & \\
\hline 1 & 3 & & & \\
\hline $\mathrm{E}$ & D & C & B & A \\
\hline
\end{tabular}

a) Even Eqilibrium Cycle

\begin{tabular}{|c|c|c|c|c|}
\hline 1 & 2 & 2 & 2 & 1 \\
\hline 2 & 3 & 2 & 1 & 3 \\
\hline 2 & 2 & 2 & 1 & \\
\hline 2 & 1 & 1 & & \\
\hline 1 & 3 & & & \\
\hline $\mathrm{E}$ & D & C & B & $A$ \\
\hline
\end{tabular}

b) Odd Equilibrium cycle 
1: Fresh assembly; 2: Reloaded assembly from N-1 cycle; 3: Reloaded assembly from N-2 cycle.

Figure 5. Loading Pattern of ACP100 (1/4 core)

Table II.: Fuel Management Design of Boron and CRs Co-controlled Core

\begin{tabular}{|l|l|}
\hline Assembly Number per Batch & $24 / 24 / 9$ \\
\hline Enrichment, \%wt & $4.95,4.45$ \\
\hline Average Enrichment of the Equilibrium Core, \%wt & 3.565 \\
\hline Loading Pattern & Partial Low Leakage \\
\hline
\end{tabular}

Table III.: FAs Usage of Boron and CRs Co-Controlled Core

\begin{tabular}{|l|l|l|l|l|l|l|l|}
\hline \multirow{2}{*}{$\begin{array}{l}\text { FA Batch } \\
\text { Serial Number }\end{array}$} & \multirow{2}{*}{$\begin{array}{l}\text { Enrichment } \\
(\% \mathrm{wt})\end{array}$} & First Cycle & Cycle 2 & Cycle3 & Cycle4 & Cycle5 & $\begin{array}{l}\text { Cycle6 } \\
\text { Equilibrium }\end{array}$ \\
\hline 1 & 1.8 & 17 & & & & & \\
\hline 2 & 3.1 & 20 & 17 & & & & \\
\hline 3 & 3.9 & 20 & 20 & 17 & & & \\
\hline 4 & 3.9 & & 8 & 8 & 1 & & \\
\hline 5 & 4.45 & & 12 & 12 & 12 & & \\
\hline 6 & 4.45 & & & 20 & 20 & 9 & \\
\hline 7 & 4.45 & & & & 16 & 16 & 1 \\
\hline 8 & 4.95 & & & & 8 & 8 & 8 \\
\hline 9 & 4.45 & & & & & 16 & 16 \\
\hline 10 & 4.95 & & & & & 8 & 8 \\
\hline 11 & 4.45 & & & & & & 16 \\
\hline 12 & 4.95 & & & & & & 8 \\
\hline
\end{tabular}

Table IV.: Fuel Management Design of Boron-Free Core

\begin{tabular}{|l|l|}
\hline Assembly Number per Batch & $\begin{array}{l}24 / 21 / 12(\text { Even Equilibrium) } \\
21 / 24 / 12(\text { Odd Equilibrium) }\end{array}$ \\
\hline Enrichment, \% & $4.95,3.9,3.1$ \\
\hline \multirow{2}{*}{ Average Enrichment of the Equilibrium Core, \%wt } & $\begin{array}{l}3.545(\text { Even Equilibrium) } \\
3.458(\text { Odd Equilibrium) }\end{array}$ \\
\hline Loading Pattern & Partial Low Leakage \\
\hline
\end{tabular}

Table V. : FAs Usage of Boron-Free Core

\begin{tabular}{|c|c|c|c|c|c|c|c|c|}
\hline \multirow{2}{*}{$\begin{array}{l}\text { FA Batch } \\
\text { Serial }\end{array}$} & \multirow{2}{*}{$\begin{array}{l}\text { Enrichment } \\
(\% w t)\end{array}$} & \multicolumn{7}{|c|}{ Amount of FAs } \\
\hline & & First & Cycle2 & Cycle3 & Cycle4 & Cycle5 & Cycle6 & Cycle7 \\
\hline
\end{tabular}




\begin{tabular}{|l|l|l|l|l|l|l|l|l|}
\hline Number & & Cycle & & & & & $\begin{array}{l}\text { Even } \\
\text { Equilibriu } \\
\mathrm{m}\end{array}$ & $\begin{array}{l}\text { Odd } \\
\text { Equilibrium }\end{array}$ \\
\hline 1 & 1.9 & 13 & 1 & & & & & \\
\hline 2 & 3.1 & 44 & 28 & 13 & & & & \\
\hline 3 & 4.95 & & 28 & 24 & 21 & & & \\
\hline 4 & 4.95 & & & 20 & 16 & 16 & & \\
\hline 5 & 4.95 & & & & 20 & 20 & 12 & \\
\hline 6 & 3.1 & & & & & 1 & 1 & \\
\hline 7 & 4.95 & & & & & 20 & 20 & 12 \\
\hline 8 & 3.9 & & & & & & 4 & 4 \\
\hline 9 & 4.95 & & & & & & 20 & 20 \\
\hline 10 & 3.1 & & & & & & & 1 \\
\hline 11 & 4.95 & & & & & & & 20 \\
\hline
\end{tabular}

\subsection{Main Results}

Table VI. shows main results of the co-controlled core, and Table VII. shows results of the boron-free core. 660 681 EFPD cycle length permits a utilization factor bigger than $90 \%$ during 24 months, reach the setting target. The main power peak factors are at the reasonable level, they have been proved to satisfy the safety and fuel limits. Both designs provide enough shutdown margin to ensure the safety. The average discharge exposure is smaller compared with large PWRs, but it is reasonably high for a small reactor. Considering that the maximum discharge exposure still has an obvious margin, there is still optimization space, especially for the co-controlled design.

Besides, for the co-controlled core, moderator feedback coefficient is $-10.65 \mathrm{pcm} /{ }^{\circ} \mathrm{C}$ in the $1^{\text {st }}$ cycle and $17.19 \mathrm{pcm} /{ }^{\circ} \mathrm{C}$ in the equilibrium cycle; for the boron-free core, moderator feedback coefficient is -47.27 $\mathrm{pcm} /{ }^{\circ} \mathrm{C}$ in the $1{ }^{\text {st }} \mathrm{cycle}-65.64 \mathrm{pcm} /{ }^{\circ} \mathrm{C}$ in the even equilibrium cycle, and $-68.79 \mathrm{pcm} /{ }^{\circ} \mathrm{C}$ in the odd equilibrium cycle. For both cores, this coefficient is negative enough.

Table VI : Main Results of Boron and CRs Co-Controlled Core

\begin{tabular}{|l|l|}
\hline Cycle Length, EFPD & 681 \\
\hline Average Discharge Exposure, MWd/tU & $\sim 40000$ \\
\hline Maximum Discharge Exposure of Assembly, MWd/tU & 47793 \\
\hline Maximum Discharge Exposure of Fuel rod, MWd/tU & 53657 \\
\hline Maximum $\mathrm{F}_{\triangle \mathrm{H}}$ & 1.44 \\
\hline Maximum $\mathrm{F}_{\mathrm{Q}}$ & 1.99 \\
\hline Shutdown Margin, pcm & $>4500$ \\
\hline
\end{tabular}

Table VII. : Main Results of Boron-Free Core

\begin{tabular}{|l|l|}
\hline Cycle Length, EFPD & 660 \\
\hline Average Discharge Exposure, MWd/tU & $\sim 42000$ \\
\hline Maximum Discharge Exposure of Assembly, MWd/tU & 50804 \\
\hline
\end{tabular}




\begin{tabular}{|l|l|}
\hline Maximum Discharge Exposure of Fuel rod, MWd/tU & 57846 \\
\hline Maximum $\mathrm{F}_{\triangle \mathrm{H}}$ & 1.60 \\
\hline Maximum $\mathrm{F}_{\mathrm{Q}}$ & 2.70 \\
\hline Maximum Keff* & 0.9895 \\
\hline
\end{tabular}

*: BOL of 1 st cycle, cold state, $0 \mathrm{Xe}$, all rods inserted with the maximum one stucked.

\section{CONCLUSIONS}

In this paper, two different core designs for Small Modular Reactor ACP100 are introduced. Both designs maximally use mature components, materials and even ideas to ensure the near-term application.

Loading pattern, reactivity control and fuel management strategy for both designs are focused in this paper. Main results are presented, 24 months cycle length target is fully achieved; key parameters are proved to satisfy the safety requirements; discharge burnup is reasonably high for SMRs.

The boron and CRs co-controlled design is more similar to large PWRs, which is suitable for land-base application. The boron-free design permits a more compact design, which can be used for sea-base applications.

\section{ACKNOWLEDGMENTS}

This paper presents nuclear design of ACP100; this work is a part of the whole ACP100 NPP design. Even though sometimes the design compromises for other related domains, but the design could never stand firmly without the whole complete design. So, thanks to the whole team who work for the reactor design of ACP 100.

\section{REFERENCES}

1. [1]Preliminary Safety Analysis Report, ACP100 Case File for IAEA Generic Reactor Safety Review (GRSR), China National Nuclear Corporation, JUNE 2015.

2. [1]Wang Liangzi, Ju Haitao, Qin Dong, etc, Fuel Management Strategy Analysis of Small Modular Pressurized Reactor ACP100, Nuclear Power Engineering, 39(1), pp. 157-160 (2018). 